\title{
The Effects of Minocycline on the Hippocampus in Lithium- Pilocarpine Induced Status Epilepticus in Rat: Relations with Microglial/Astrocytic Activation and Serum S100B Level
}

\author{
Erkut Baha BULDUK ${ }^{1}$, Gokhan KURT'1 , Sureyya BARUN², Onder AYDEMIR ${ }^{3}$, Murat KIZILTAS ${ }^{2}$, Murat OKTEM ${ }^{4}$, \\ Turan TURHAN ${ }^{5}$, Pergin ATILLA ${ }^{6}$, Sevda MUFTUOGLU 6
}

${ }^{1}$ Gazi University, Faculty of Medicine, Department of Neurosurgery, Ankara, Turkey

${ }^{2}$ Gazi University, Faculty of Medicine, Department of Pharmacology, Ankara, Turkey

${ }^{3}$ Gazi University, Faculty of Medicine, Department of Public Health, Ankara, Turkey

${ }^{4}$ Duzen Laboratories Group, Department of Biochemistry, Ankara, Turkey

${ }^{5}$ Ankara Numune Education and Research Hospital, Department of Biochemistry, Ankara, Turkey

${ }^{6}$ Hacettepe University Medical School, Department of Histology, Ankara, Turkey

\section{ABSTRACT}

AIM: To investigate possible correlations between serum S100B levels and microglial/astrocytic activation in status epilepticus (SE) in lithium-pilocarpine-exposed rat hippocampi and whether serum S100B levels linearly reflect neuroinflammation. Additionally, to assess the effects of minocycline (M), an inhibitor of neuroinflammation.

MATERIAL and METHODS: Rats were divided into 4 groups (6/group), namely, control (C), sham, SE, and SE+M. Animals were exposed to lithium-pilocarpine to induce SE in the SE and SE+M groups. Cardiac blood was collected to measure S100B levels, and coronal brain sections including the hippocampus were prepared to examine microglial/astrocytic activation and to evaluate neuroinflammation at day 7 of SE.

RESULTS: Serum S100B levels, OX42 (+) microglia in CA1, and GFAP (+) astrocytes in both CA1 and dentate gyrus (DG) were higher in the SE+M group than in the $\mathrm{C}$ group. Most importantly, highly positive correlations were found between S100B levels and microglial activation in CA1, apart from astrocytic activation in CA1 and DG. Unexpectedly, microglial activation in CA1 and astrocytic activation in DG were also enhanced in the SE+M group compared with the $\mathrm{C}$ group. Moreover, $\mathrm{M}$ administration reversed the neuronal loss observed in DG during SE.

CONCLUSION: These results suggest that serum S100B is a candidate biomarker for monitoring neuroinflammation and that it may also help predict diagnosis and prognosis.

KEYWORDS: S100B, Astrocyte, Microglia, Minocycline, Seizure

\section{INTRODUCTION}

$\mathrm{E}$ pilepsy is a chronic neurological disorder characterized by recurrent seizures resulting from uncontrollable neural excitation in the brain (28), and status epilepticus (SE) is associated with an increased risk of developing epilepsy $(25,26)$. Although seizures and epilepsy are primarily considered neuronal diseases, studies have shown that pathogenesis may be associated with non-neuronal components (e.g., neuroinflammation) $(23,27)$.

Neuroinflammation appears to be one of the etiological factors that promote epileptogenesis (57). Contrastingly, seizure activity induced by various methods can cause inflammation 
in the brain through the transcription of inflammatory genes $(14,56)$. A previous study has shown that the frequency of seizures is positively correlated with the levels of inflammation in the affected tissue in epileptic patients (47). Moreover, anti-inflammatory treatments lead to anticonvulsant effects in patients with epileptic seizures who are refractory to classical antiepileptic drugs (58). Neuroinflammation is also implicated as a causative factor in neurodegeneration resulting from seizures (2), and activation of astrocytes and microglia is associated with neuroinflammatory processes (60). Thus, it has been suggested that neuroinflammation is not merely an epiphenomenon but that it may actively contribute to the pathophysiology of epilepsy.

$\mathrm{S} 100 \mathrm{~B}$ is a member of the multigenic family of $\mathrm{Ca} 2+-$ binding proteins of the EF-hand type and is prominently expressed in astroglial cells (24). Its action in the brain is both as an intracellular regulator and extracellular signal. Intracellular $\mathrm{S} 100 \mathrm{~B}$ acts as a stimulator of cell proliferation and migration and inhibits apoptosis. The activation of astrocytes may contribute to repair after brain damage (16), and astrocytes constitutively release $\mathrm{S} 100 \mathrm{~B}$ into the extracellular space, which then acts on neurons, astrocytes, and microglia via its primary receptor, namely, receptor for advanced glycation end products $(15,44)$.

As an extracellular factor, low concentrations (nM) of S100B promote neuronal survival, whereas higher concentrations $(\mu \mathrm{M})$ result in harmful events, including brain inflammation and neuronal apoptosis (16). Microglial cells are activated by S100B under pathological conditions and release cytokines (1). Thus, serum S100B levels are elevated in central nervous system (CNS) pathologies, including trauma, stroke, inflammatory diseases, psychiatric conditions, and seizures $(35,65)$; conversely, normal serum S100B levels reliably predict the absence of significant CNS injury (3).

Minocycline $(M)$ is a second-generation tetracycline antibiotic that readily crosses the blood-brain barrier (BBB) and exhibits anti-inflammatory, antiapoptotic, and neuroprotective effects independent of its antimicrobial action $(40,67)$. Therefore, it has been used to evaluate the inhibition of inflammation under various CNS pathologies $(48,68,69)$.

It is known that neuroinflammatory processes contribute to seizure pathophysiology and that $\mathrm{S100B}$ levels increase due to epileptic seizures $(29,35,49)$. As $S 100 B$ may enter the bloodstream, S100B has been proposed as a biomarker for brain damage (32). However, it remains unknown whether S100B levels linearly reflect neuroinflammatory processes, including microglial/astrocytic activation and neuronal loss, after seizures. If true, S100B levels can be used for predicting the following: 1) contribution of neuroinflammation to pathophysiology, 2) efficiency of treatment, and 3) prognosis. Further, if indeed there is a correlation between these parameters, S100B may be useful as a biomarker. To date, any potential associations among neuroinflammation, neuronal loss, and serum S100B levels have not been investigated in SE. Given the above, we hypothesized that $\mathrm{S100B}$, an astrocytic protein, elevated as a result of various neurological insults, is a biomarker of neuroinflammation after lithium-pilocarpin-induced SE and that its level will reduce as a result of $\mathrm{M}$ treatment.

Thus, we aimed to assess any possible correlations among microglial/astrocytic activation, neuronal loss, and serum S100B levels in a rat model of SE. Additionally, the antiinflammatory effect of $M$ was used to observe any change in these parameters. Microglial/astrocytic activation was investigated by analyzing glial fibrillary acidic protein (GFAP) and OX 42 expressions, respectively $(22,62)$. Further, brain tissue $\mathrm{S100B}$ expression was not assessed because its expression is not considered a marker of microglial/astrocytic activation.

\section{MATERIAL and METHODS}

\section{Animals}

In total, 24 male Sprague Dawley rats weighing 200-250 g, were used. The animals were housed in a facility with a 12hour artificial light-dark cycle and allowed free access to a standard diet and tap water. All experiments were conducted according to the ethical standards in Directive 86/609/EEC. This study was approved by the Gazi University Ethics Committee for Animals (2012/45-03).

\section{Experimental Protocol}

Animals were divided into 4 groups:

1) Control group (C),

2) Sham group (S),

3) SE group,

4) $M+S E$ group.

Each group included 6 animals. In the $C$ group, all rats received an identical volume of saline $(0.9 \% \mathrm{NaCl}$, i.p. $)$ as treated animals, instead of the chemicals. Animals in the $S$ group were treated identically to those in the SE group except for pilocarpine administration. The animals received lithium, methylscopolamine, and diazepam. However, saline was administered instead of pilocarpine.

\section{Induction of SE using Lithium-Pilocarpine}

Rats were pretreated with lithium chloride $(127 \mathrm{mg} / \mathrm{kg}$, i.p.) before pilocarpine injections (30 mg/kg, i.p.) as described previously (30). Seizures were graded according to the Racine score (46). Continuous limbic seizures corresponding to classes 4-5 were defined as SE. In this study, rats exhibited seizures in a few minutes following the initial pilocarpine injection, which corresponded to classes 1-3 in the SE and $\mathrm{SE}+\mathrm{M}$ groups (46). Therefore, pilocarpine was reinjected at 30 minutes following the first injection to induce SE, and all rats administered the second pilocarpine injection remained in SE until termination with diazepam. To the best of our knowledge, this is the first experimental protocol for lithium-pilocarpineinduced SE. Because it has been previously shown that SE lasting for 30-60 minutes is sufficient to induce hippocampal damage (9), diazepam was administered $(10 \mathrm{mg} / \mathrm{kg}$, i.p.) to terminate the seizures 1 hour after the first pilocarpine injec- 
tion. If the first dose of diazepam was not effective, injections ( $5 \mathrm{mg} / \mathrm{kg}$, i.p.) were repeated till the seizures stopped or for a maximum of 3 times. Additionally, methylscopolamine (1 $\mathrm{mg} / \mathrm{kg}$, i.p.) was administered 30 minutes before the first pilocarpine injection to reduce peripheral cholinergic side effects of pilocarpine (e.g., salivation, diarrhea, body tremors, and chromodacryorrhea). All rats exposed to lithium-pilocarpine were fed water-soaked food until they were able to eat normal, dry food pellets. Rats were also administered $0.9 \%$ saline (10 $\mathrm{mL}$ per animal) subcutaneously to restore volume loss. All the experiments were conducted at the same time and in the morning. As pre-insult delivery of $M$, followed by post-insult administration, exhibits the greatest improvement after brain injury, we also employed this strategy (50). In the SE+M group, the first $M$ injection $(40 \mathrm{mg} / \mathrm{kg}$, i.p.) was administered 1 hour before the lithium chloride injection; this was repeated for 7 days. Considering that astrocytic and neuroplastic changes occur immediately following pilocarpine-induced seizures, animals were sacrificed after 7 days of SE, and the brains were removed to analyze astroglial and microglial damage $(50,51)$. Intracardiac blood was collected from rats for estimating serum S100B levels before removing the brain.

\section{Histological Examination}

All animals were anesthetized using thiopental ( $30 \mathrm{mg} / \mathrm{kg}$, i.p.) and were sacrificed. Brains were removed and put on ice. The area including the hippocampus was removed from the rest of the brain by coronal dissections, both in the front and back. After fixation in $10 \%$ buffered formaldehyde for 72 hours, the hippocampus tissue was routinely processed for parafinization, $5-\mu \mathrm{m}$-thick coronal serial sections cut on a microtome, and every 1 in 5 sections $(1,14,28,40,60)$ stained with hematoxylin-eosin (HE). GFAP immunohistochemistry was performed on 2 consecutive sections $(16,24,25,27,35,47,56,65,68,69)$ for assessing astrocyte activation, whereas OX-42 immunohistochemistry was performed on the next 2 consecutive sections $(2,3,15,23,44,48,49,57,58,67)$ for estimating microglial cell activation. GFAP immunoreactive cells and OX-42 immunoreactive cells were evaluated both in the dentate gyrus (DG) and CA1 regions in each section. Immunoreactive cells in 3 areas in each section were counted independently by 2 histologists blinded to the identity of the samples. The mean values for both were taken for each section, and the mean values for each antibody were calculated in each experimental group. Pycnotic neurons were also counted by the same method in the HE-stained sections for each experimental group.

\section{Assessment of Serum S100B Levels}

Intracardiac blood samples $(3 \mathrm{ml})$ were collected from rats and centrifuged at $2000 \mathrm{rpm}$ for 10 minutes; serum was collected and stored at $-80^{\circ} \mathrm{C}$ until analysis. Commercially available ELISA kits were used according to the manufacturer's protocols (SunRed Biotechnology Lot No. 201502).

\section{Chemicals}

Lithium chloride, methylscopolamine, pilocarpine, thiopental, and diazepam were purchased from Sigma-Aldrich (Steinheim, Germany).

\section{Statistical Analyses}

Data were evaluated by Statistical Package for Social Sciences (SPSS) for Windows 15.0 (SPSS Inc, Chicago, IL). Descriptive statistics are presented as median (minimum-maximum). The Kruskal-Wallis test was used to compare more than 3 groups, and the post-hoc Bonferroni correction was used to estimate statistical significance. Statistical significance level for Type-I error was set at $5 \%(p<0.05)$. Correlations were evaluated using the Spearman correlation coefficient.

\section{RESULTS}

Experimental observations of adult rats subjected to lithiumpilocarpine-induced SE. Classes 1-3 seizures were observed in all 24 rats within a few minutes after the first pilocarpine injection, and classes 4-5 seizures, which correspond to SE, were observed in all rats following the second pilocarpine injection; these intensive seizures lasted 30 minutes until termination with diazepam (46).

Most previously published studies indicate that most rats experience SE recurrence in the 90-120-min period following SE termination using antiseizure drugs (4). Contrastingly, we found that diazepam injection terminates seizures in all animals with no recurrence during the 90-120-min period. However, it must be noted that the animals were not monitored by electroencephalography (EEG) but were only observed during the experiments. Further and favorably, other studies report that lithium-pilocarpine-induced SE for 60 min does not lead to epilepsy during a subsequent 6-month period (5). Scopolamine can inhibit SE when administered before pilocarpine but is not effective after SE has been established (7). Because methylscopolamine cannot cross the BBB, it is used to reduce the peripheral muscarinic adverse effects of pilocarpine (8). However, methylscopolamine may have crossed the BBB due to greater permeability after neuroinflammation, thereby possibly contributing to the inhibition of recurrence in our study (10-12). Lastly, it is also possible that this effect is specific to this model.

Of the 6 rats in the SE+M group, 2 died, yielding a mortality rate of $36 \%$. Therefore, experiments were repeated with 2 animals and the data combined to maintain 6 animals per group. No deaths were observed in the other groups.

\section{Serum S100B Levels}

Significant differences in serum S100B levels were found among the experimental groups $(p=0.008)$, and comparisons between 2 groups showed that SE and M+SE groups had higher S100B levels than the $C$ group $(p=0.008$ and $p=0.002$, respectively; Figure 1).

\section{Immunohistochemical Evaluation}

\section{Evaluation of OX 42(+) microglia in CA1}

There was a significant difference in OX 42(+) microglia in CA1 in the experimental groups $(p=0.012)$ and OX 42(+) microglia were significantly higher in the $\mathrm{SE}$ and $\mathrm{SE}+\mathrm{M}$ groups than the $C$ and $S$ groups $(p=0.004, p=0.008, p=0.002$, and $p=0.008$, respectively; Figure 2). 
Evaluation of OX 42(+) microglia in DG

There was no statistically significant difference in cell numbers among the groups $(p=0.277$; Figure 3$)$.

\section{Evaluation of GFAP $(+)$ astrocytes in CA1}

Significant differences in GFAP $(+)$ astrocytes in CA1 were found among the experimental groups $(p=0.012)$. GFAP $(+)$ astrocyte cell numbers in $\mathrm{CA} 1$ were significantly higher in the $S E+M$ group than the $C$ and $S$ groups $(p=0.008$ and $p=0.003$, respectively; Figure 4).

Evaluation of GFAP $(+)$ astrocytes in DG

GFAP (+) astrocyte numbers in DG were significantly different

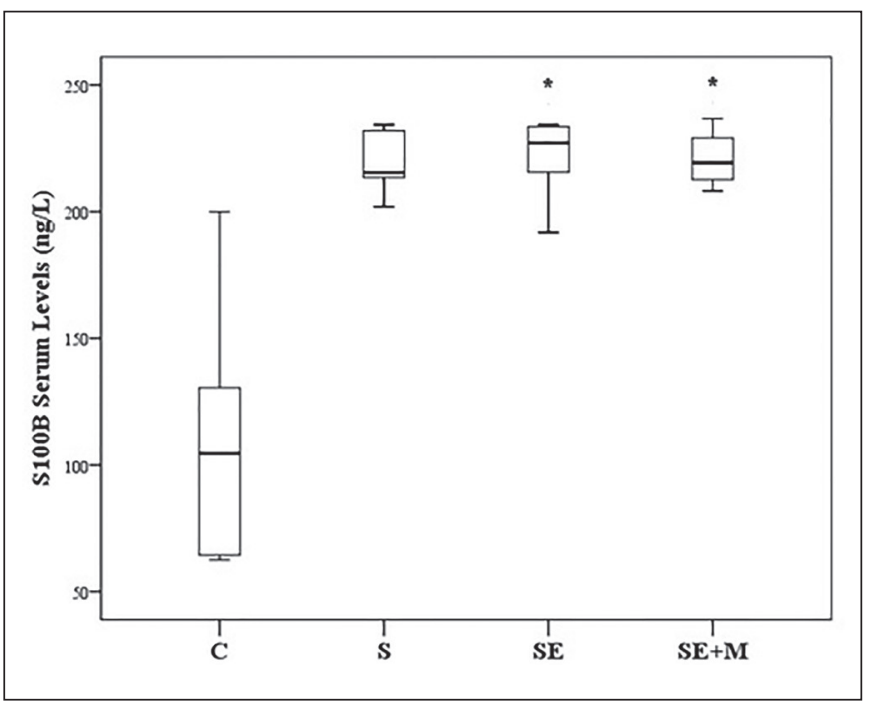

Figure 1: S100B levels in the experimental groups. Data are the median (range) of 6 experiments. ${ }^{*} p<0.05$ compared with $C$.

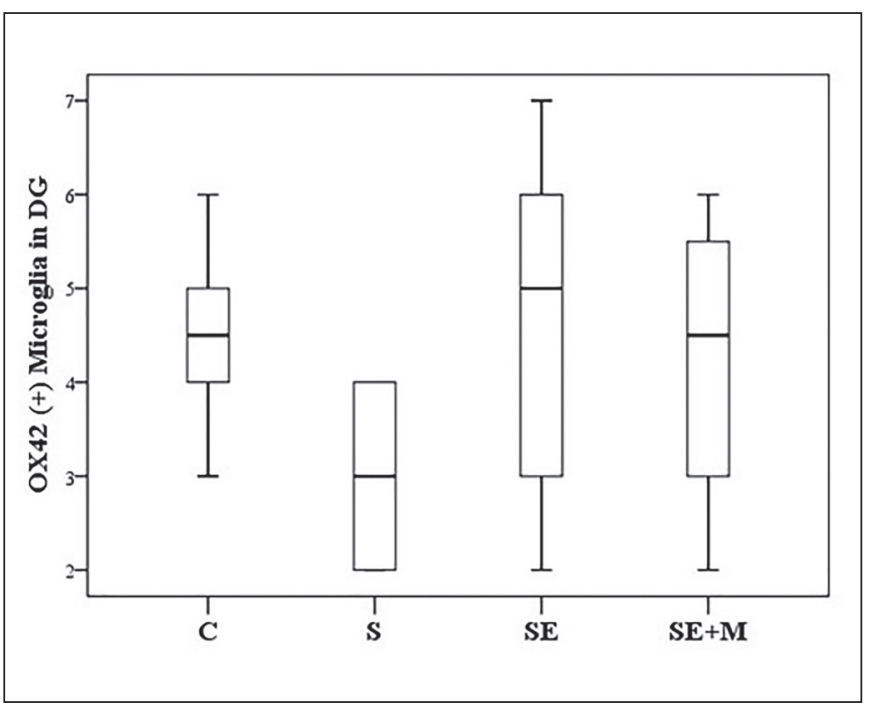

Figure 3: OX 42(+) microglia in DG in the experimental groups. Data are the median (range) of 6 experiments. among the experimental groups $(p=0.007)$, with higher cell numbers in the $S E$ and $S E+M$ groups than the $C$ and $S$ groups $(p=0.001, p=0.004, p=0.005$, and $p=0.008$, respectively; Figure 5).

Correlation between S100B levels and OX 42(+) microglia and GFAP (+) astroglia in the experimental groups

There was no correlation between these parameters in the $\mathrm{C}$ group.

In the $S$ group, a negative correlation was found between the S100B level and GFAP (+) astroglia in CA1 in SE $(r=-0.90)$ (Figure 6).

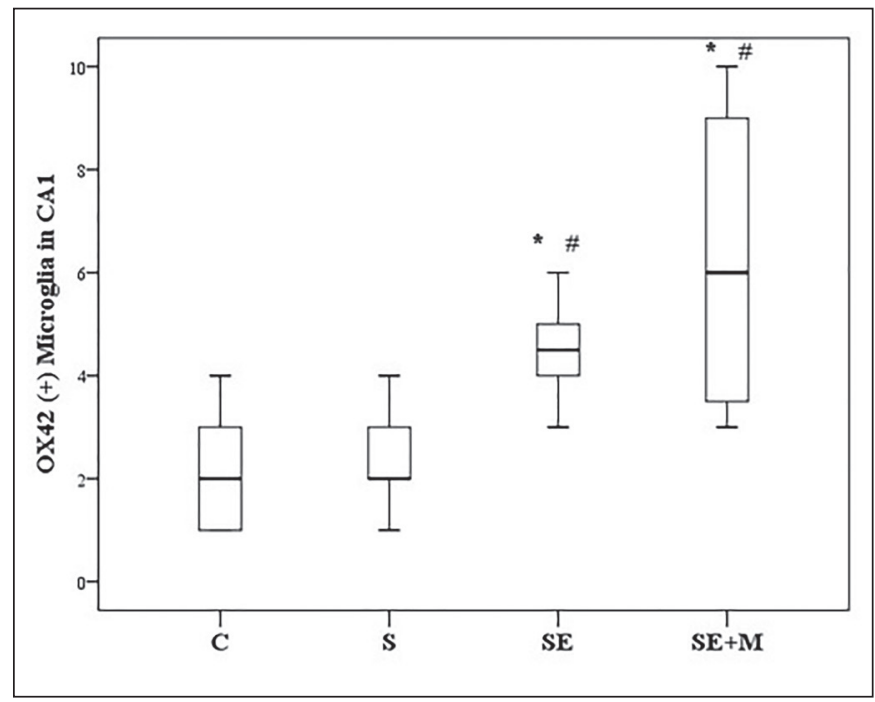

Figure 2: OX 42(+) microglia in CA1 in the experimental groups. Data are the median (range) of 6 experiments. ${ }^{*} p<0.05$ compared with $\mathrm{C}$; $\# \mathrm{p}<0.05$ compared with $\mathrm{S}$.

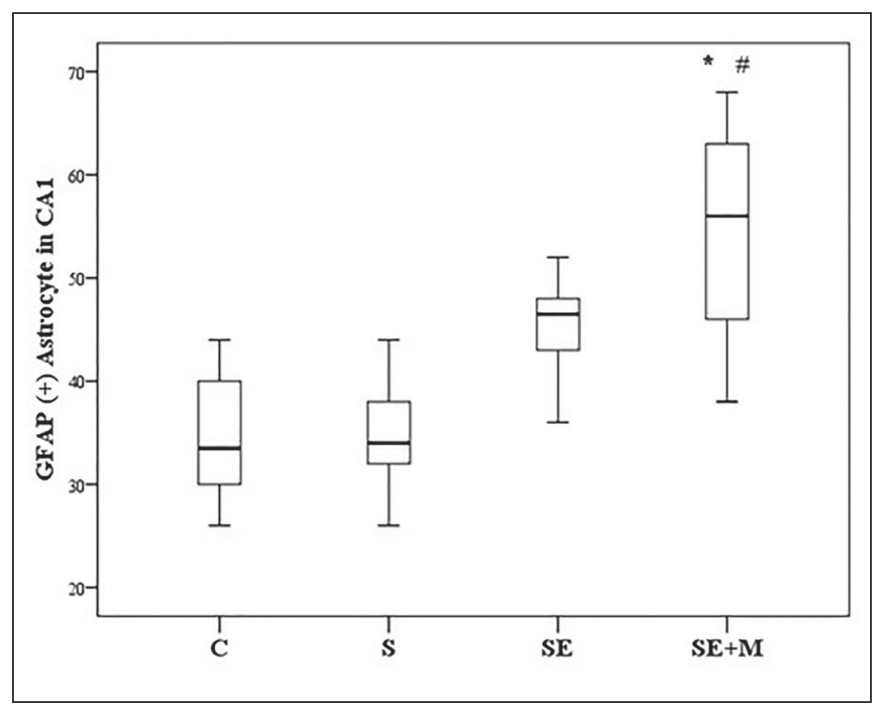

Figure 4: GFAP (+) astrocytes in CA1 in the experimental groups. Data are the median (range) of six experiments. ${ }^{*} \mathrm{p}<0.05$ compared with $\mathrm{C} ; \# \mathrm{p}<0.05$ compared with $\mathrm{S}$. 
In the SE group, the following results were obtained:

1) GFAP (+) astrocytes in CA1 and DG showed a significant positive correlation ( $r=0.94$; Figure 7)

2) a positive correlation was found between $S 100 B$ levels and OX 42(+) microglia in CA1 ( $r=0.82$; Figure 8).

No correlation was observed between S100B levels and OX 42(+) microglia. S100B levels were positively correlated with GFAP (+) astrocytes in CA1 and DG ( $r=0.94$ and $r=0.83$, respectively; Figures 9, 10).

No correlation between the parameters tested was found in the M+SE group (Table I).

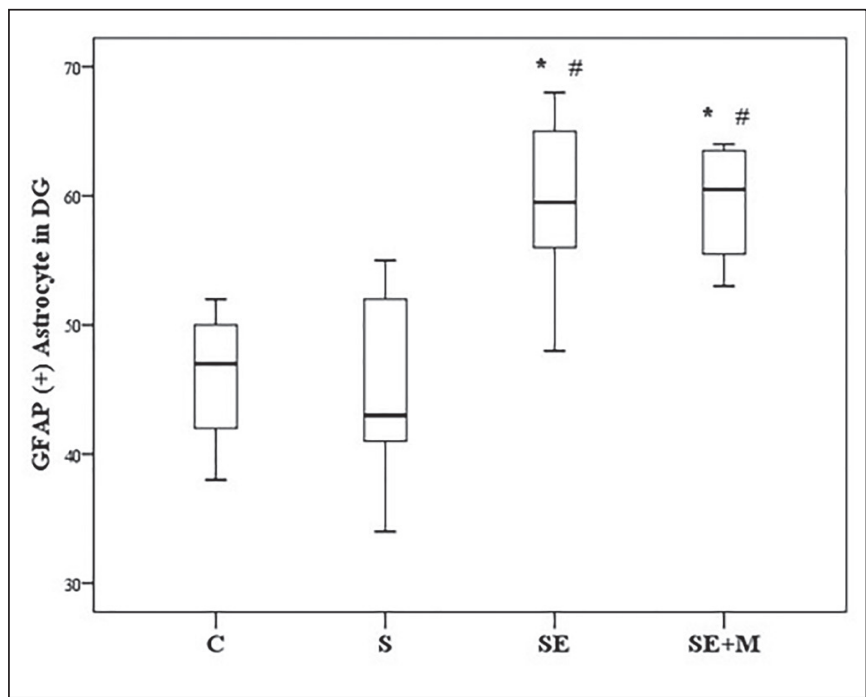

Figure 5: GFAP (+) astrocytes in DG in the experimental groups. Data are the median (range) of 6 experiments. ${ }^{*} p<0.05$ compared with $\mathrm{C} ; \# \mathrm{p}<0.05$ compared with $\mathrm{S}$.

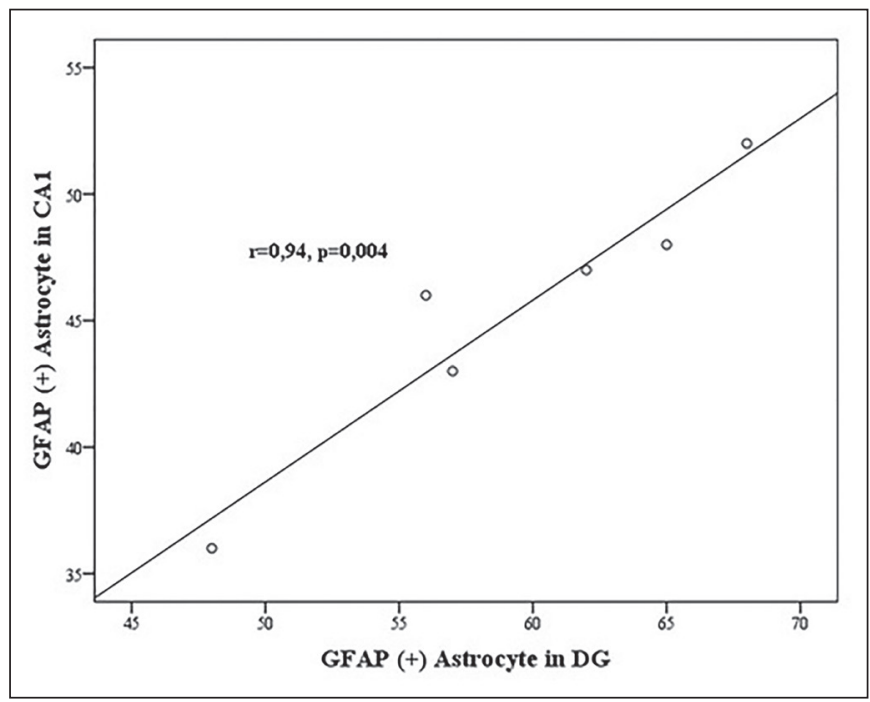

Figure 7: Positive correlation between GFAP(+) astrocytes in DG and CA1 in the SE group.

\section{Histological Evaluation}

Group C

Microscopic examination showed normal morphology of DG and CA1. GFAP (+) astroglia and weak OX 42(+) immunoreactivity were also detected in DG and CA1 (Figures 11A, B; 12A, B, respectively).

\section{Groups S and SE}

Histological appearances are not presented for these groups. Data are given in Figures 2-5.

\section{Group M+SE}

GFAP (+) astroglia were prominent in DG and CA1 in this group

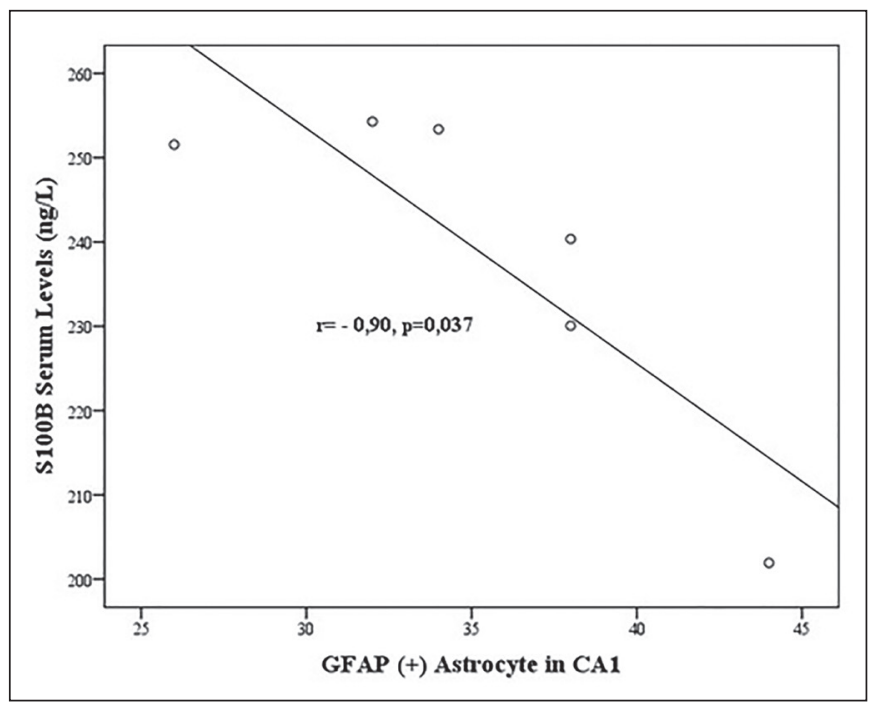

Figure 6: Negative correlation between S100B levels and GFAP $(+)$ astrocytes in CA1 in the S group.

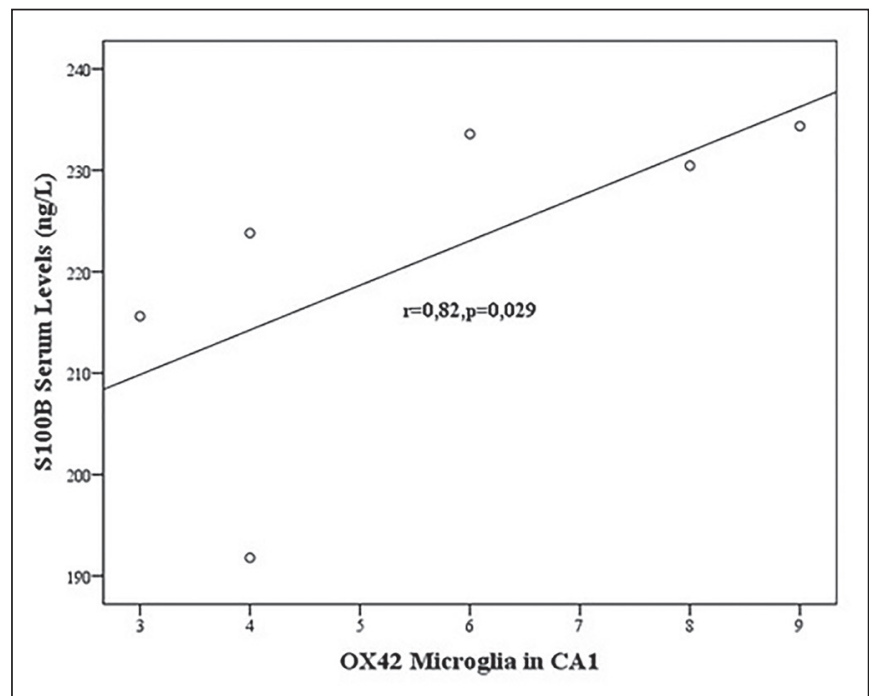

Figure 8: Positive correlation between S100B levels and OX 42(+) microglia in CA1 in the SE group. 
Bulduk EB. et al: Minocycline's Effect in Microglial Activation

Table I: Investigation of the Correlation Between S100B Level and Microglial/Astrocytic Activation in SE+M Group

\begin{tabular}{|c|c|c|c|c|c|c|}
\hline$S E+M$ & & GFAP (+) DG & GFAP (+) CA1 & OX42 (+) DG & OX42 (+) CA1 & S100B \\
\hline \multirow{2}{*}{ GFAP (+) DG } & $\mathbf{r}$ & 1.000 & & & & \\
\hline & p & ----- & & & & \\
\hline \multirow{2}{*}{ GFAP (+) CA1 } & $r$ & -0.200 & 1.000 & & & \\
\hline & p & 0.800 & ---- & & & \\
\hline \multirow{2}{*}{ OX42 (+) DG } & $r$ & 0.001 & -0.400 & 1.000 & & \\
\hline & $\mathbf{p}$ & 0.998 & 0.600 & ----- & & \\
\hline \multirow{2}{*}{ OX42 (+) CA1 } & $r$ & -0.001 & 0.200 & 0.001 & 1.000 & \\
\hline & $\mathbf{p}$ & 0.998 & 0.800 & 0.998 & ----- & \\
\hline \multirow{2}{*}{ S100B } & $r$ & 0.400 & -0.800 & -0.200 & -0.400 & 1.000 \\
\hline & $\mathbf{p}$ & 0.600 & 0.200 & 0.800 & 0.600 & ----- \\
\hline
\end{tabular}

*r: Spearman correlation coefficient.

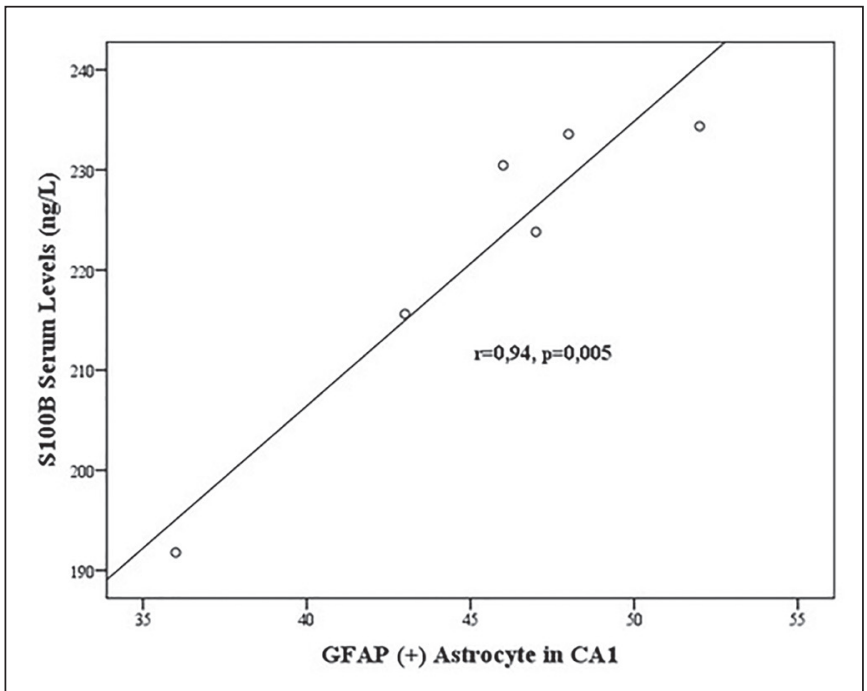

Figure 9: Positive correlation between S100B levels and GFAP(+) astrocytes in CA1 in the SE group.

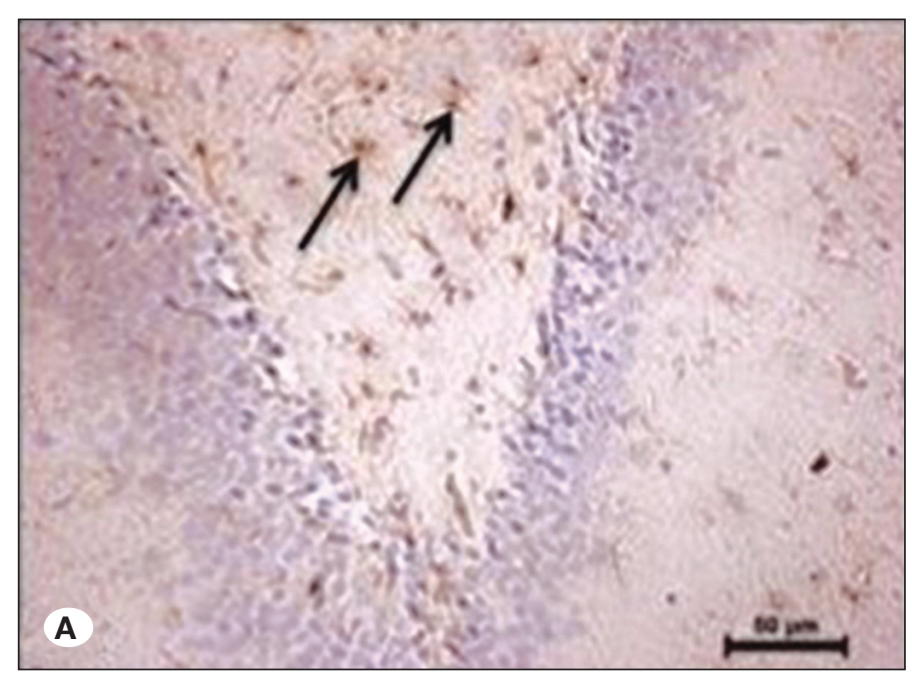

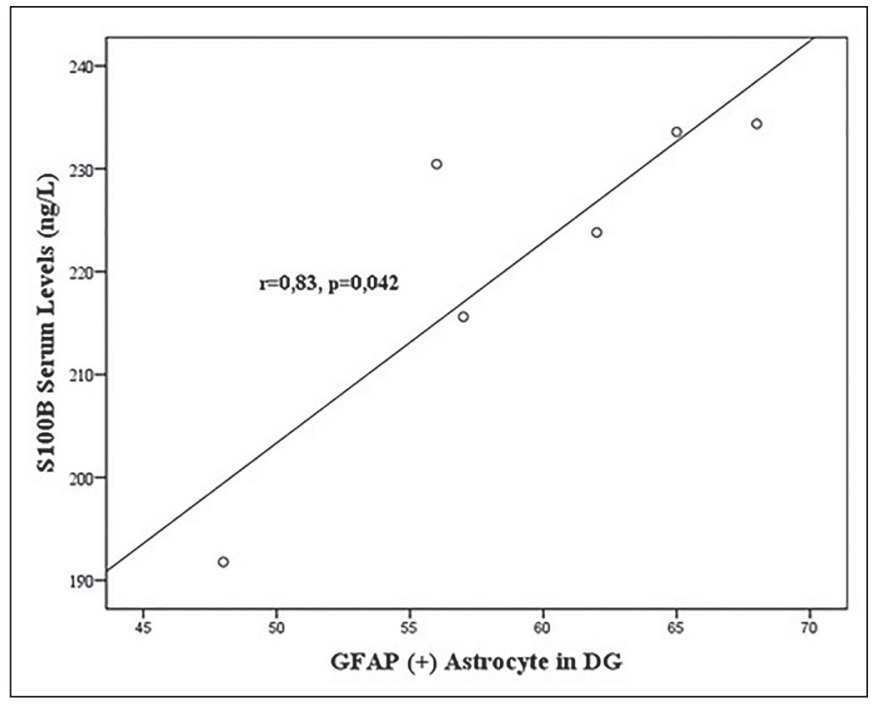

Figure 10: Positive correlation between S100B levels and GFAP (+) astrocytes in DG in the SE group.

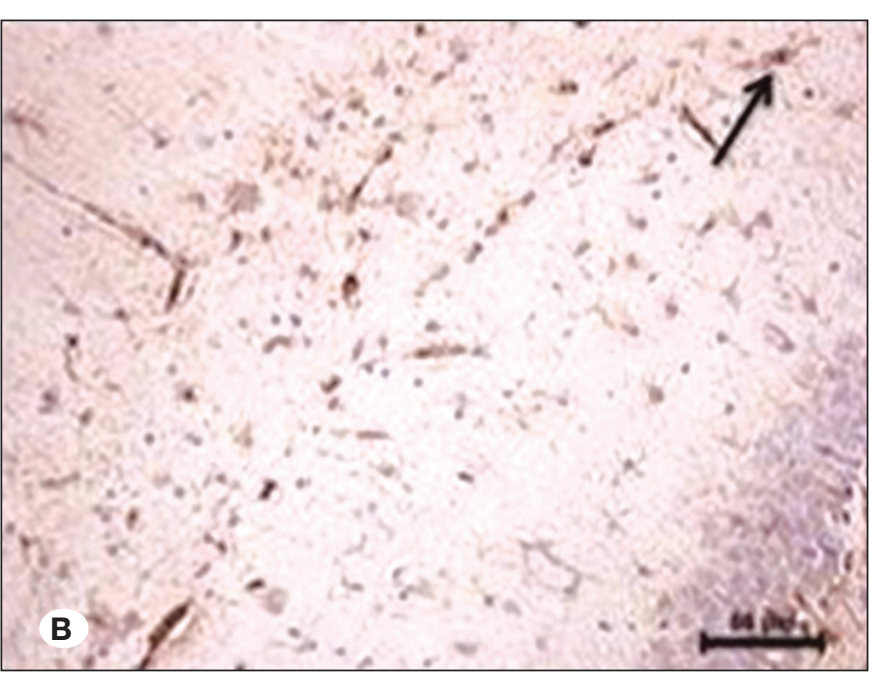

Figure 11: GFAP (+) astrocytes in DG (A), and CA1 (B) in the C group (HRP/ABC-hematoxylin-eosin staining; $\mathbf{A}: \times 200, \mathbf{B}: \times 200)$. 


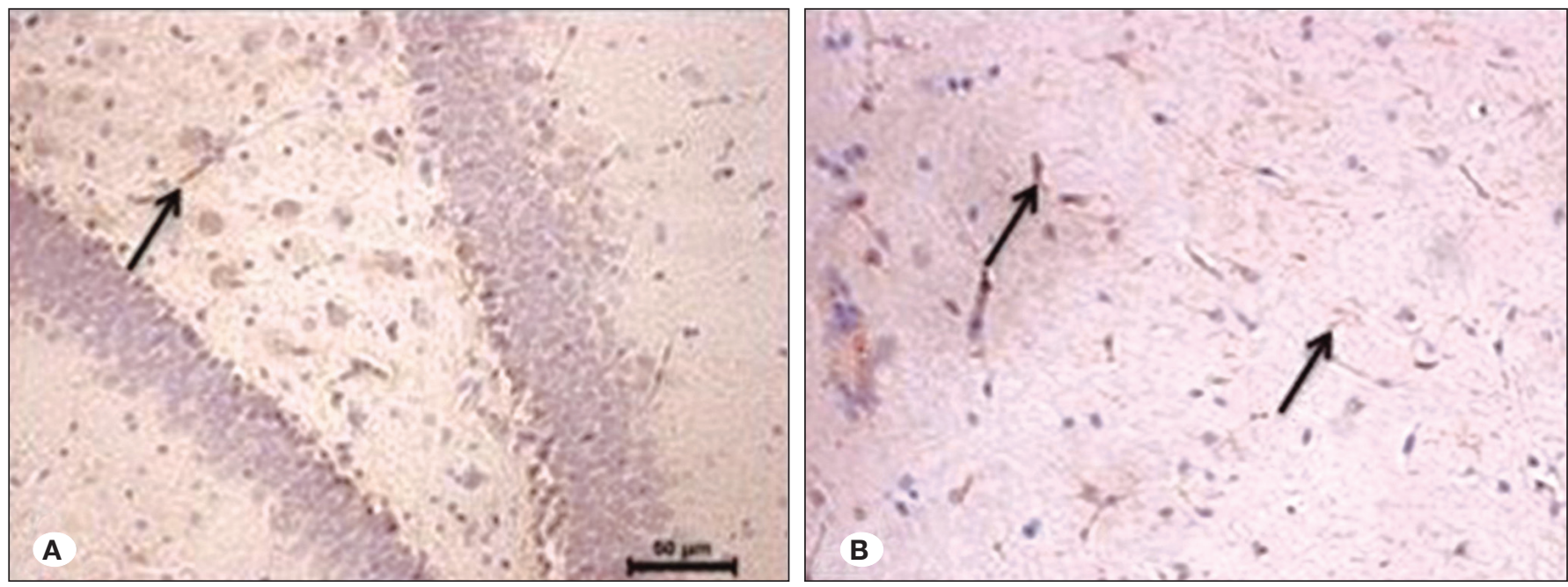

Figure 12: OX 42(+) microglia in DG (A), and CA1 (B) in the C group (HRP/ABC-hematoxylin-eosin staining; A: $\times 200$, B: $\times 200)$.
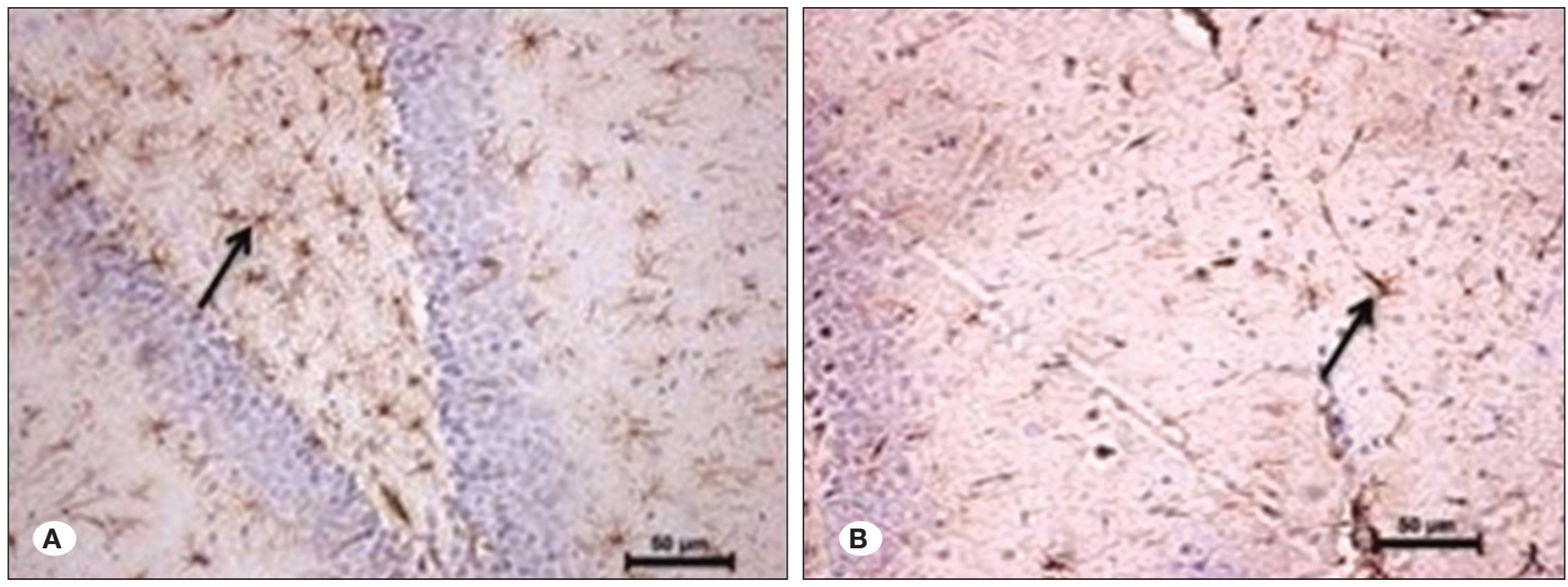

Figure 13: GFAP (+) astrocytes in DG (A), and CA1 (B) in the M+SE group (HRP/ABC-hematoxylin-eosin staining; A: $\times 200, \mathbf{B}: \times 200)$.
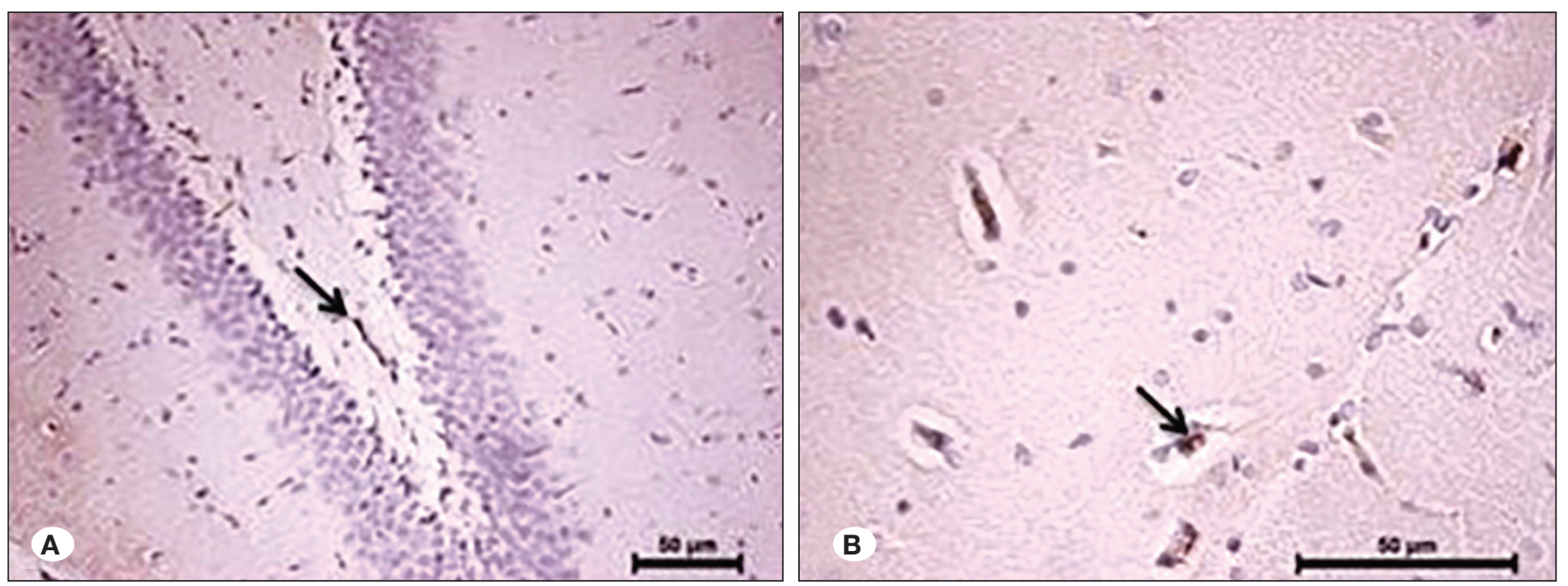

Figure 14: OX42 (+) microglia in DG (A), and CA1 (B) in the M+SE group. 
Table II: Pycnotic Neurons in DG and CA1 in Experimental Groups

\begin{tabular}{|c|c|c|c|c|c|}
\hline & $\mathbf{n}$ & DG & $\mathbf{p}^{*}$ & CA1 & $\mathbf{p}^{*}$ \\
\hline C & 6 & 55 (34-66) & \multirow{3}{*}{$0.001^{a}$} & $4.5(3-9)$ & \multirow{3}{*}{0.127} \\
\hline $\mathbf{S}$ & 6 & $71(52-98)$ & & $8(4-11)$ & \\
\hline SE & 6 & $129(110-165)$ & & $8.5(5-13)$ & \\
\hline
\end{tabular}

*Kruskal-Wallis Test

a Significant difference between SE and C, and SE and SE+M.

(Figures 13A, B). Additionally, OX $42(+)$ microglial cells were observed in both DG and CA1 (Figure 14A, B).

Pycnotic neurons were counted, and data are presented in Table II.

Necrotic neurons were found to be positively correlated with OX 42(+) microglia $(r=0.58)$ in CA1 in SE.

\section{DISCUSSION}

$\mathrm{S} 100 \mathrm{~B}$ is expressed predominantly in astrocytes and plays a critical role in neuroinflammation (10), and reactive astroglia overexpress GFAP and secrete S100B after seizures $(23,27,45)$. Additionally, S100B stimulates microglial activation and leads to the release of interleukin- 6 and interleukin $1 a(52,63)$. Further, neuroinflammation is crucial in neuronal loss in the CNS. The activation of Toll-like receptors (TLRs) expressed in the microglia causes injury to neurons (33). Research and clinical data have indicated that seizures are associated with elevated S100B protein levels in both serum and cerebrospinal fluid (CSF) $(35,37)$. However, it remains unknown whether serum S100B levels linearly reflect neuroinflammatory changes and neuronal loss in seizures.

We showed that serum S100B levels are significantly higher after lithium-pilocarpine-induced SE, and other studies have shown that S100B levels are elevated in CSF in SE in an identical animal model (13). Moreover, S100B levels in serum and CSF are increasingly being used as biomarkers of brain damage and neurodegenerative diseases to determine the extent of neuronal damage (65). Thus, in agreement with other published results, the elevated S100B levels in SE observed in our study also appear to originate from the activation of astrocytes, which was in turn due to seizure-induced excitotoxicity $(12,38,49)$. S100B is released from astrocytes through the activation of the metabotrophic glutamate receptor 3 (49). In line with this view, it has been found that mGlu2 and 3 receptor expressions are increased in the hippocampi of patients with temporal lobe epilepsy (55).

GFAP (+) astrocytes in DG after SE were significantly higher than those of controls. However, astrocytic activation was not different in CA1 between the SE and C groups. Nevertheless, a highly positive correlation was observed between GFAP (+) astrocytes in both DG and CA1 ( $r=0.94)$ in SE. Taken together, these results suggest that GFAP (+) astrocytes in DG and CA1 increase in tandem, but astrocyte activation in CA1 did not reach statistical significance.

To the best of our knowledge, this is the first report on possible correlations between serum S100B levels and microglial/ astrocytic activation in CA1 and DG. The major findings are that S100B levels are positively correlated with GFAP (+) astrocyte numbers both in DG and CA1 ( $r=0.83$ and $r=0.94$, respectively), suggesting that serum $S 100 B$ levels probably linearly reflect astrocyte activation both in DG and CA1.

OX42 (+) microglial cells in DG were not different among the 4 experimental groups, implying that DG is not markedly involved in microglial activation. Further supporting this result is the observation that there is no correlation between OX 42 (+) microglia in DG and S100B levels.

In CA1, OX 42(+) microglia were significantly higher in the SE group than the $\mathrm{C}$ group. Furthermore, OX $42(+)$ microglia were positively correlated with significantly high levels of S100B $(r=0.82)$, and previous studies have also reported similar results $(39,53)$. Consistent with this result, other reports have demonstrated that $\mathrm{S} 100 \mathrm{~B}$ released from activated astrocytes stimulates microglial activation $(52,63)$.

Taken together, it appears that serum S100B levels linearly reflect microglial activation in CA1 and astrocytic activation in both CA1 and DG. These results are important for predicting the degree of neuroinflammatory processes by measuring serum S100B levels. Additionally, astrocytes may release cytotoxic factors and glial cell line-derived neurotrophic factor in the presence of activated microglia (7). Therefore, our data also provide important insights regarding neuronal loss. Necrotic neurons were found to be positively correlated with OX 42(+) microglia in CA1 in SE ( $r=0.58)$, indicating a prominent cytotoxic effect of microglia in CA1. Previous studies have suggested that neuroinflammation is a driving force for neurodegeneration (21). The activation of TLRs expressed on microglia leads to neuronal injury (33). Additionally, the synergistic effects of S100B with interferon gamma in stimulating nitric oxide secretion in microglia may have also contributed to the observed neurotoxicity in CA1 in SE $(1,43)$.

Another aspect that required consideration in this study was that microglial/astrocytic activation are ongoing processes on day 7 following SE induction. Likewise, the activation of 
microglia has been shown to continue for at least 3-5 days following pilocarpine-induced SE (51). Therefore, parallel elevation in S100B levels is an expected result in our study.

In the M-treated group, S100B levels were higher than those of the $\mathrm{C}$ group. Unexpectedly, $\mathrm{M}$ also led to an increase in OX 42(+) microglia in CA1 and GFAP (+) astrocytes, both in DG and CA1, compared with the $C$ group. These results do not concur with those of previous studies, which demonstrate an anti-inflammatory effect of $M(8,20,42,61)$. However, there was no correlation between S100B levels and microglial/astrocytic activation in both DG and CA1 in rats exposed to SE in the presence of $\mathrm{M}$. Taken together, these results imply that $\mathrm{M}$ does not attenuate SE-induced microglial/astrocytic activation. It is possible that this lack of correlation between these parameters in M-treated rats is due to the limited inflammatory capacity of the tissue. Moreover, neuroinflammation leads to greater BBB permeability (34). Therefore, another explanation for the observed lack of correlation may be that the inhibitory action of $M$ on the increased permeability of BBB obscured any positive correlation between S100B levels and microglial/ astrocytic activation in the hippocampus. In support of this hypothesis, other studies have shown that $\mathrm{M}$ can alleviate BBB permeability loss through various mechanisms $(19,41,64)$ and that serum and brain S100B levels are poorly correlated, with serum levels dependent primarily on the integrity of BBB and not on the level of S100B in the brain (31).

Herein, $M$ reversed neuronal necrosis observed in CA1 in $\mathrm{SE}$. It has been reported that both astrocytic and microglial activation precedes neuronal damage (30). Thus, it is feasible to expect that $\mathrm{M}$, a microglia inhibitor, would attenuate the harmful effects of seizures such as neuronal necrosis. However, $M$ reversed the neuronal necrosis observed in DG in SE but without a demonstrable anti-inflammatory effect, probably implying that the neuroprotective effect of $M$ is not anti-inflammatory-related. In fact, the anti-inflammatory effects were not clearly observable in our study. In line with our results, but apart from its anti-inflammatory actions, $\mathrm{M}$ has previously been shown to have neuroprotective properties and could reduce neuronal necrosis $(5,18,20,66)$. Additionally, it was suggested that it is possible for $M$ to prevent an increase in inflammatory cytokines without affecting their inactivate state (8). Other neuroprotective mechanisms may include antiapoptotic protein upregulation and oxidative stress counteraction $(6,59)$.

The $S$ group, although not statistically different from the C group, showed an increase in S100B levels. Additionally, a negative correlation was found between $\mathrm{S} 100 \mathrm{~B}$ levels and GFAP (+) astrocytes. This effect may have been due to the inhibitory effect of lithium on microglial activation (17). Furthermore, scopoloamine reduces the toxicity of glutamate receptor agonists, like kainate and domoic acid $(36,54)$. Therefore, it is unclear if these results can be reproduced in other experimental SE models. Thus, future studies need to obtain additional information to evaluate this experimental animal model.

\section{CONCLUSION}

SE led to an increase in S100B levels and caused the persistent activation of microglia and astrocytes. These findings show that S100B levels are highly and positively correlated with OX42 (+) microglia in CA1 and GFAP (+) astrocytes in both CA1 and DG. Additionally, SE-induced neuronal necrosis in DG could be reversed by $M$ without an accompanying antiinflammatory effect. S100B levels were also found to be higher in M-administered animals than in controls. Nevertheless, there was no correlation between S100B levels and microglial/ astrocytic activation. Neuroinflammation and the search for biomarkers are currently the focus of epileptogenesis research. The translational aspect of this study is that the $100 \mathrm{~B}$ protein may be a candidate biomarker for evaluating the relationship between neuroinflammation and epileptogenesis. Predicting neuroinflammatory alterations in the presence or absence of drug treatment, or during preoperative or postoperative periods of epilepsy surgery, will enhance our understanding of the pathophysiology of epilepsy and will also help in designing new treatment approaches. Additionally, biomarkers may be useful as cost-effective tools for screening potential antiepileptogenic drugs.

\section{ACKNOWLEDGMENT}

This study was supported by Gazi University Scientific Research Projects Unit with 01/2014-08 project code and received the Turkish Neurosurgical Society Prof. Dr. Aysima Altınok Thesis Award for the Specialization of Neurosurgery in 2016. The sponsor had no role in the design or conduct of this research. Preparation for publication of this article is partly supported by Turkish Neurosurgical Society.

\section{- REFERENCES}

1. Adami C, Sorci G, Blasi E, Agneletti AL, Bistoni F, Donato R: S100B expression in and effects on microglia. Glia 33: 131142, 2001

2. Allan SM, Rothwell NJ: Cytokines and acute neurodegeneration. Nat Rev Neurosci 2: 734-744, 2001

3. Bloomfield SM, McKinney J, Smith L, Brisman J: Reliability of S100B in predicting severity of central nervous system injury. Neurocrit Care 6: 121-138, 2007

4. Brandt C, Tollner K, Klee R, Broer S, Löscher W: Effective termination of status epilepticus by rational pharmacology in the lithium-pilocarpine model in rats: Window of opportunity to prevent epilepsy and prediction of epilepsy by biomarkers. Neurobiol Dis 75: 78-90, 2015

5. Buller KM, Carty ML, Reinebrant HE, Wixey JA: Minocycline: A neuroprotective agent for hypoxic-ischemic brain injury in the neonate? J Neurosci Res 87: 599-608, 2009

6. Cai Z, LinS, Fan L-W, Pang Y, Rhodes PG: Minocyclinealleviates hypoxic ischemic injury to developing oligodendrocytes in the neonatal rat brain. Neuroscience 137: 425-435, 2006

7. Chen SH, Oyarbazal EA, Sung YF, Chu CH, Wang Q, Chen SL, Lu RB, Hong JS: Microglial regulation of immunological and neuroprotective functions of astroglia. Glia 63: 118-131, 2015 
8. Cheng S, Hou J, Zhang C, Xu C, Wang L, Zou X, Yu H, Shi Y, Yin Z, Chen G: Minocycline reduces neuroinflammation but does not ameliorate neuron loss in a mouse model of neurodegeneration. Sci Rep 5: 105-135, 2015

9. Chu K, Jung KH, Lee ST, Kim JH, Kang KM, Kim HK, Lim JS, Park HK, Kim M, Lee SK, Roh JK: Erythropoietin reduces epileptogenic processes following status epilepticus. Epilepsia 49: 1723-1732, 2008

10. Craft JM, Watterson DM, Marks A, Van Eldik LJ: Enhanced susceptibility of S-100B transgenic mice to neuroinflammation and neuronal dysfunction induced by intracerebraventricular infusion of human $\beta$-amyloid. Glia 51: 209-216, 2005

11. Curia G, Longo D, Biagini G, Jones RS, Avoli M: The pilocarpine model of temporal lobe epilepsy. J Neurosci Methods 172: 143-157, 2008

12. De Bock F, Dornand J, Rondouin G: Release of TNF alpha in the rat hippocampus following epileptic seizures and excitotoxic neuronal damage. Neuroreport 7: 1125-1129, 1996

13. De Oliveira DL, Fischer A, Jorge RS, Da Silva MC, Leite M, Goncalves CA, Quillfeldt JA, Souza DO, E Souza TM, Wofchuk $\mathrm{S}$ : Effects of early-life LiCl-pilocarpine-induced status epilepticus on memory and axiety in adult rats are associated with mossy fiber sprouting and elevated CSF S100B protein. Epilepsia 49: 842-852, 2008

14. De Simoni MG, Perego C, Ravizza T, Moneta D, Conti M, Marchesi F, De Luigi A, Garattini S, Vezzani A: Inflammatory cytokines and related genes are induced in the rat hippocampus by limbic status epilepticus. Eur J Neurosci 12: 2623-2633, 2000

15. Donato R: RAGE: A single receptor for several ligands and different cellular responses: The case of certain $\mathrm{S} 100$ proteins. Curr Mol Med 7: 711-724, 2007

16. Donato R, Sorci G, Riuzzi F, Arcuri C, Bianchi R, Brozzi F, Tubaro C, Giambanco I: S100B's double life: Intracellular regulator and extracellular signal. Biochim Biophys Acta 1793: 1008-1022, 2009

17. Dong H, Zhang X, Dai X, Lu S, Gui B, Jin W, Zhang S, Zhang S, Qian Y: Lithium ameliorates lipopolysaccharide-induced microglial activation via inhibition of toll-like receptor 4 expression by activating the P13K/Akt/Fox01 pathway. J Neuroinflammation 11: 140, 2014

18. Elewa HF, Hilali H, Hess DC, Machado LS, Fagan S: Minocycline for short-term neuroprotection. Pharmacotherapy 26: 515-521, 2006

19. Engelhardt S, Patkar S, Ogunshola OO: Cell-specific bloodbrain barrier regulation in health and disease: A focus on hypoxia. Br J Pharmacol 171: 1210-1230, 2014

20. Fan L-W, Lin S, Pang Y, Rhodes PG, Cai Z: Minocycline attenuates hypoxia-ischemia-induced neurological dysfunction and brain injury in the juvenile rat. Eur J Neurosci 24: 341-350, 2006

21. Gao HM, Hong J: Why neurodegenerative diseases are progressive: uncontrolled inflammation drives disease progression. Trends Immunol 29: 357-365, 2008

22. Garrison CJ, Dougherty PM, Kajander KC, Carlton SM: Staining of glial fibrillary acidic protein (GFAP) in lumbar spinal cord increases following a sciatic nerve constriction injury. Brain Res 565: 1-7, 1991
23. Garzillo CL, Mello LE: Characterization of reactive astrocytes in the chronic phase of the pilocarpine model of epilepsy. Epilepsia 5: 107-109, 2002

24. Heizmann CW, Fritz G, Schaefer BW: S100 proteins: Structure, functions and pathology. Front Biosci 7: d1356-d1368, 2002

25. Hesdorffer DC, Logroscino G, Gascino G, Annegers JF, Hauser WA: Risk of unprovoked seizure after acute symptomatic seizure: Effect of status epilepticus. Ann Neurol 44: 908-912, 1998

26. Imran I, Hillert MH, Klein J: Early metabolic responses to lithium/pilocarpine-induced status epilepticus in rat brain. $J$ Neurochem 135: 1007-1018, 2015

27. Jankowsky JL, Patterson PH: The role of cytokines and growth factors in seizures and their sequelae. Prog Neurobiol 6: 125-149, 2001

28. Jefferys JG: Models and mechanisms of experimental epilepsies. Epilepsia 12: 44-50, 2003

29. Kacinski M, Budziszewska B, Lason W, Zajac A, SkowronekBala B, Leskiewicz M, Kubik A, Basta-Kaim A: Level of S100B protein, neuron specific enolase, orexin $A$, adiponectin and insulin-like growth factor in serum of pediatric patients suffering from sleep disorders with or without epilepsy. Pharmacol Rep 64: 1427-1433, 2012

30. Kang TC, Kim DS, Kwak SE, Kim JE, Won MH, Kim DW, Choi SY, Kwon OS: Epileptogenic roles of astroglial death and regeneration in the dentate gyrus of experimental temporal lobe epilepsy. Glia 54: 258-271, 2006

31. Kleindienst A, Ross Bullock M: A critical analysis of the role of the neurotrophic protein $S 100 B$ in acute brain injury. $J$ Neurotrauma 23: 1185-1200, 2006

32. Kleindienst A, Schmidt C, Parsch H, Emtmann I, Xu Y, Buchfelder M: The passage of S100B from brain to blood is not specifically related to the blood-brain barrier integrity. Cardiovasc Psychiatry Neurol 2010: 801295, 2010

33. Lehnardt $S$, Massillon L, Follet $P$, Jensen FE, Ratan $R$, Rosenberg PA, Volpe JJ, Vartanian T: Activation of innate immunity in the CNS triggers neurodegeneration through a Toll-like receptors 4-dependent pathway. Proc Natl Acad Sci U S A 100: 8514-8519, 2003

34. Li YJ, Wang ZH, Zhang B, Zhe X, Wang MJ, Shi ST, Bai J, Lin T, Guo CJ, Zhang SJ, Kong XL, Zuo X, Zhao H: Disruption of the blood-brain barrier after generalized tonic-clonic seizures correlates with cerebrospinal fluid MMP-9 levels. J Neuroinflammation 10: 80, 2013

35. Lu C, Li J, Sun W, Feng L, Li L, Liu A, Li J, Mao W, Wei H, Gao L, Zhang X, Huang Z, Meng X, Wang Y: Elevated plasma $\mathrm{S} 100 \mathrm{~B}$ concentration is associated with mesial temporal lobe epilepsy in Han Chinese: A case- control study. Neurosci Lett 484: 139-142, 2010

36. McGeer EG, McGeer PL: Substantia nigra cell death from kainic acid or folic acid injections into the pontine tegmentum. Brain Res 298: 339-342, 1984

37. Meng XJ, Wang F, Li CK: Resveratrol is neuroprotective and improves cognition in pentylenetetrazole-kindling model of epilepsy in rats. Indian J Pharm Sci 72: 125-131, 2014

38. Mori T, Koyama N, Arendash GW, Horikoshi-Sakubara Y, Tan J, Town T: Overexpression of human S100B exacerbates cerebral amyloidosis and gliosis in Tg2576 mouse model of Alzheimer's disease. Glia 58: 300-314, 2010 
39. Mrak RE, Griffin WST: The role of activated astrocytes and of the neurotrophic cytokine S100B in the pathogenesis of Alzheimer's disease. Neurobiol Aging 22: 915-922, 2001

40. Noble W, Garwood CJ, Hanger DP: Minocycline is a potential therapeutic agent in neurodegenerative disorders characterized by protein misfolding. Prion 3: 78-83, 2009

41. Ogunshola OO, Al-Ahmad A: HIF-1 at the blood-brain barrier: A mediator of permeability? High Alt Med Biol 13: 153-161, 2012

42. Parachikova A, Vasilevko V, Cribbs DH, LaFerla FM, Gree KN: Reductions in amyloid-beta-derived neuroinflammation, with minocycline, restore cognition but do not significantly affect tau hyperphosphorylation. J Alzheimer Dis 21: 527-572, 2010

43. Petrova T, Hu J, Van Eldik LJ: Modulation of glial activation by astrocyte-derived protein S100B: Differential responses of astrocyte and microglial cultures. Brain Res 853: 74-80, 2000

44. Pinto SS, Gottfried C, Mendez A, Goncalves D, Karl J, Goncalves CA, Wofchuk S, Rodnight R: Immunocontent and secretion S100B in astrocyte cultures from different brain regions in relation to morphology. FEBS Letters 486: 203-207, 2000

45. Quincozes-Santos A, Rosa RB, Leipnitz G, de Souza DF, Seminotti B, Wajner M, Gonçalves CA: Induction of S100B secretion in $\mathrm{C} 6$ astroglial cells by the major metabolites accumulating in glutaric acidemia type I. Metab Brain Dis 25: 191-198, 2010

46. Racine R, Okujava V, Chipashvili S: Modification of seizure activity by electrical stimulation. 3. Mechanisms. Electroencephalogr Clin Neurophysiol 32: 295-299, 1972

47. Ravizza T, Boer K, Redeker S, Spliet WG, van Rijen PC, Troost D, Vezzani A, Aronica E: The IL-beta system in epilepsyassociated malformations of cortical development. Neurobiol Dis 24: 128-143, 2006

48. Ryu JK, Franciosi S, Sattayaprasertv P, Kim Su, McLarnon JG: Minocycline inhibits neuronal death and glial activation induced by beta-amyloid peptide in rat hippocampus. Glia 48: 85-90, 2004

49. Sakatani S, Seto-Ohshima A, Shinohara $Y$, Yamamato $H$, Yamamato S, Itohara H, Hirase S: Neural-activity dependent release of S100B from astrocytes enhances kainate-induced gamma oscillations in vivo. J Neurosci 28: 10928-10936, 2008

50. Shapiro LA, Figueroa-Aragon S, Ribaki CE: Newly generated granule cells show rapid neuroplastic changes in the adult rat dentate gyrus during the first five days following pilocarpineinduced seizures. Eur J Neurosci 26: 583-592, 2007

51. Shapiro LA, Wang L, Ribak CE: Rapid astrocyte and microglial activation following pilocarpine-induced seizures in rats. Epilepsia 49: 33-41, 2008

52. Sheng JG, Mrak RE, Griffin WS: Neuritic plaque evolution in Alzheimer's disease is accompanied by transition of activated microglia from primed to enlarged to phagocytic forms. Acta Neuropathol 94: 1-5, 1997

53. Sheng JG, Mrak RE, Griffin WS: S100 beta protein expression in Alzheimer disease: Potential role in the pathogenesis of neuritic plaques. J Neurosci Res 39: 398-404, 1994

54. Sobotka TJ, Brown R, Quander DY, Jackson R, Smith M, Long SA, Barton CN, Rountree RL, Halls S, Eilers P, Johannessen JN, Scallet AC: Domoic acid: Neurobehavioral and neurohistological effects of low-dose exposure in adult rats. Neurotoxicol Teratol 18: 659-670, 1996
55. Tang FR, Lee WL, Yeo TT: Expression of the group I metabotropic glutamate receptor in the hippocampus of patients with mesial temporal lobe epilepsy. J Neurocytol 30: 403-411, 2001

56. Turrin NP, Rivest S: Innate immune reaction in response to seizures: implications for the neuropathology associated with epilepsy. Neurobiol Dis 16: 321-334, 2004

57. Vezzani A, Balosso S, Ravizza T: Inflammation and epilepsy. Handb Clin Neurol 107: 163-175, 2012

58. Vezzani A, Granata T: Brain inflammation in epilepsy. Experimental and clinical evidence. Epilepsia 46: 1724-1743, 2005

59. Wang J, Wei Q, Wang CY, Hill WD, Hess DC, Dong Z: Minocycline up-regulates $\mathrm{Bcl}-2$ and protects against cell death in mitochondria. J Biol Chem 279: 19948-19954, 2004

60. Wetherington J, Serrano G, Dingledine R: Astrocytes in the epileptic brain. Neuron 58: 168-178, 2008

61. Wu DC, Jackson-Lewis V, Vila M, Tieku K, Teismann P, Vadsth C, Choi DK, Ischiropoulos H, Przedborski S: Blockade of microglial activation is neuroprotective in the 1-methyl-4phenyl-1,2,3,6-tetrahydropyridine mouse model of Parkinson disease. J Neurosci 22: 1763-1771, 2002

62. Yamamoto $\mathrm{Y}$, Terayama $\mathrm{R}$, Kishimoto $\mathrm{N}$, Maruhama $\mathrm{K}$, Mizutani M, lida S, Sugimoto T: Activated microglia contribute to convergent nociceptive inputs to spinal dorsal horn neurons and the development of neuropathic pain. Neurochem Res 40: 1000-1012, 2015

63. Yan SD, Chen X, Fu J, Chen M, Zhu H, Roher A, Slattery T, Zhao L, Nagashima M, Morser J, Migheli A, Nawroth P, Stern D, Schmidt AM: RAGE and amyloid-beta peptide neurotoxicity in Alzheimer's disease. Nature 382: 685-691, 1996

64. Yang F, Zhou L, Wang D, Wang Z, Huang QY: Minocycline ameliorates hypoxia-induced blood-brain barrier damage by inhibition of HIF-1a through SIRT-3/PHD-2 degradation pathway. Neuroscience 304: 250-259, 2015

65. Yardan T, Erenler AK, Baydin A, Aydin K, Cokluk C: Usefulness of S100B protein in neurological disorders. J Pak Med Assoc 61: 276-281, 2011

66. Yenari MA, Xu L, Tang XN, Qiao Y, Giffard RG: Microglia potentiate damage to blood-brain barrier constituents: Improvement by minocycline in vivo and in vitro. Stroke 37: 1087-1093, 2006

67. Yong VW, Wells J, Giuliani F, Casha S, Power C, Metz LM: The promise of minocycline in neurology. Lancet Neurol 3: 744751,2004

68. Yrjanheikki J, Keinanen R, Pellikka M, Hokfelt T, Koistinaho $\mathrm{J}$ : Tetracyclines inhibit microglial activation and are neuroprotective in global brain ischemia. Proc Natl Acad Sci 22: 15769-15774, 1998

69. Yrjanheikki J, Tikka T, Keinanen R, Golsteins G, Chan PH, Koistinaho JA: Tetracycline derivative, minocycline, reduces inflammation and protects against focal cerebral ischemia with a wide therapeutic window. Proc Natl Acad Sci 96: 13496-13500, 1999 